\title{
THE PARTITION FUNCTION FOR TOPOLOGICAL FIELD THEORIES
}

\author{
by \\ J. Gegenberg ${ }^{\dagger}$ G. Kunstatter ${ }^{\sharp}$ \\ ${ }^{\dagger}$ Dept. of Mathematics and Statistics, University of New Brunswick \\ Fredericton, New Brunswick \\ Canada E3B 5 A3 \\ \# Winnipeg Institute for Theoretical Physics and Physics Dept. \\ University of Winnipeg \\ Winnipeg, Manitoba \\ Canada R3B 2E9
}

\begin{abstract}
We use a Hodge decomposition and its generalization to non-abelian flat vector bundles to calculate the partition function for abelian and non- abelian BF theories in $n$ dimensions. This enables us to provide a simple proof that the partition function is related to the Ray-Singer torsion defined on flat vector bundles for all odd-dimensional manifolds, and is equal to unity for even dimensions.
\end{abstract}

June 1992, revised March 1993

WIN-92-03

UNB Technical Report 92-01 


\section{INTRODUCTION}

Topological field theories, such as Einstein gravity in $2+1$ spacetime dimensions or the so-called BF theories $[2,11,12,13]$ are characterized by the following properties: First, the gauge group is sufficiently large so as to eliminate all local dynamics. Second, physical quantities are independent of the choice of spacetime metric. The net result is that the construction of quantum versions of these theories proceeds much like the construction of quantum mechanical systems[4,11].

Topological field theories are of interest for at least two important reasons. First they provide examples of quantum field theories in which the non-perturbative sector can be examined quantitatively. It is therefore hoped that these simpler theories will yield insight into the non-perturbative quantization of more complicated, and physically relevant theories. An important example in this regard is quantum gravity. A complete quantum gravity theory in $3+1$ spacetime dimensions does not yet exist, despite considerable recent progress by Ashtekar and his group (for a recent review, see Reference 3) in the canonical quantization. Einstein gravity in $2+1$ spacetime dimensions, on the other hand, is a topological field theory which is completely solvable, both classically and quantum mechanically $[4,5,6,7,8]$. From the latter, some progress has been made in solving important technical problems(e.g. the construction of observables[9]) and in sorting out deep conceptual issues(e.g., the nature of time[10]).

Second, topological field theories have provided a new framework for understanding important topological invariants. To cite two examples, we mention first the discovery by Horowitz and Srednicki[12] and by Blau and Thompson[2] that the linking number of two surfaces can be represented by a correlation function computed with respect to a quantized abelian BF theory; and second, Witten's representation of knot polynomials as expectation values of Wilson loop observables with respect to quantized non-abelian Chern-Simons theories[14].

The partition function is an important tool for topological quantum field theory, as well as for traditional quantum field theory. In the latter it plays a key part in the determination of the perturbative structure, while in the former it yields important topological invariants $[2,13,14,15]$. The most serious impediment to the calculation of the partition function (and of course other functional integrals) is the degeneracy of the lagrangian density due to the gauge invariance of the action, and in topological field theories, as in traditional quantum field theories, the technique employed is some variant of the FaddeevPopov ansatz[16]. Schwarz[13] used a method (dubbed the "method of resolvents") based on the latter to compute the partition function for abelian BF theory, in which the fields 
reside in a vector bundle with a given flat connection, in terms of the Ray-Singer torsion associated with that flat connection. However, Horowitz and Srednicki[12] computed correlation functions for the same class of theories as Schwarz by simply parameterizing the fields by their Hodge decomposition. There was no need to gauge fix since the Hodge decomposition explicitly parameterizes the gauge and the physical degrees of freedom, and the Jacobian of the transformation to the latter can be read off from the inner product.

In their review of topological field theories, Blau and Thompson[2] note that the Fadeev-Popov ansatz applied to the computation of the partition function for the abelian BF models amounts to factoring out and cancelling the integral over the exact parts and functionally integrating over the co-exact parts of the Hodge decomposition of the fields. This provides them with a preferred gauge choice, which they then use to compute the partition function via the BRST procedure.

The above discussion suggests the use of the Hodge decomposition of the fields as a parameterization in which the partition function for topological field theories can be directly calculated without encountering the usual complications that arise in the BRST procedure. The purpose of this paper is to show that this suggestion can be "rigorously" implemented for abelian Chern-Simons and for both abelian and non-abelian BF theories in arbitrary dimensions. As expected, in the abelian case the technique reproduces the results of Schwarz[13]. However, in the non-abelian case, the method allows us to write down for the first time, to our knowledge, the partition functions for non-abelian BF theory in $n$-dimensions. In particular we are able to prove the triviality of the partition function in even dimensions, and in odd dimensions to relate the partition function to a sum over Ray-Singer torsions associated with the equivalence class of flat connections parametrized by the moduli space of the theories.

The paper is organized as follows: Section 2. reviews the Hodge decomposition and evaluates the partition function for abelian Chern-Simons and $n$-dimensional BF theory. Section 3. presents the generalization of these methods to non-abelian BF theories. The proofs of some important identities are relegated to an Appendix. Section 4. closes with a summary and discussion. 


\section{ABELIAN THEORIES}

In this Section, it is shown that a Hodge decomposition allows one to compute functional integrals for abelian BF and Chern-Simons models in a straightforward manner. This method is equivalent to Schwarz' method of resolvents[2,13] for abelian BF models. Similar techniques have been used by Horowitz and Srednicki[12] to compute linking numbers.

\subsection{The Parametrization}

First, let us recall the Hodge decomposition of a differential form[17]. Given a smooth $p$-form $\omega_{(p)}$ on a closed compact orientable differentiable manifold $M$ of dimension $n \geq p$, then there exist smooth differential forms $\alpha_{(p-1)}, \beta_{(p+1)}, h_{(p)}$ such that

$$
\omega_{(p)}=h_{(p)}+d \alpha_{(p-1)}+\delta \beta_{(p+1)}
$$

where $d$ denotes exterior differentiation, $\delta \equiv(-1)^{n p+n+1} * d *$, with $*$ denoting the Hodge dual. Note that $* * \omega_{(p)}=(-1)^{p(n-p)} \omega_{(p)}$. The manifold $M$ must be provided with a metric tensor in order to define Hodge duality, co-derivatives and Laplacians[18]. In (2.1) $h_{(p)}$ is harmonic, i.e.,

$$
d h_{(p)}=0=\delta h_{(p)},
$$

so that

$$
\Delta_{(p)} h_{(p)}:=(d \delta+\delta d) h_{(p)}=0 .
$$

The exterior derivative $d$ and the corresponding co-derivative $\delta$ are nilpotent: $d d=0=\delta \delta$. The terms $d \alpha_{(p-1)}$ and $\delta \beta_{(p+1)}$ are called the exact and co-exact parts, respectively, by mathematicians; or the longitudinal (or pure gauge) and transverse parts, respectively, by physicists. It is important that the harmonic, longitudinal and transverse parts of the $\omega_{(p)}$ are unique, providing that we exclude zero modes of the operators $(\delta d)$ and $(d \delta)$ from the set of forms $\alpha_{(p-1)}$ and $\beta_{(p+1)}$, respectively. Moreover, we require that the forms $\alpha_{(p-1)}$ and $\beta_{(p+1)}$ are themselves uniquely specified and that they can be explicitly represented. This in turn can be achieved by requiring $\alpha_{(p-1)}$ and $\beta_{(p+1)}$ to be transverse and longitudinal, respectively. That is, if we consider the Hodge decomposition of $\alpha_{(p-1)}$, we find that the longitudinal part of $\omega_{(p)}$, namely $d \alpha_{(p-1)}$ is equal to $d \delta \gamma_{(p)}$, where $\delta \gamma_{(p)}$ is the unique transverse part of $\alpha_{(p-1)}$. Hence we can parametrize $\alpha_{(p-1)}$ by its unique transverse part. This parametrization can be made explicit by expanding $\gamma_{(p)}$ in terms of the eigenmodes (excluding zero modes) of the operator $d \delta$. Similarly we can parametrize $\beta_{(p+1)}$ by its unique longitudinal part. 
The harmonic part $h_{(p)}$ depends only on the global structure, i.e. the topology, of $M$. Indeed, for a given $p$, the space of harmonic $p$-forms is a vector space isomorphic to the de Rham cohomology of $M[17,18]$.

In the following discussion it will be necessary to define a functional measure on the space of $p$-forms. This can be done by first noting the existence of a natural inner product between any two $p$-forms, $\omega_{(p)}$ and $\omega_{(p)}^{\prime}[12]$ :

$$
<\omega_{(p)} \mid \omega_{(p)}^{\prime}>:=\int_{M} \omega_{(p)} \wedge * \omega_{(p)}^{\prime} .
$$

This leads to a natural functional measure $\mathcal{D} \mu\left[\omega_{(p)}\right]$ on the space of $p$-forms, namely that which normalizes the Gaussian functional integral:

$$
\int \mathcal{D} \mu\left[\omega_{(p)}\right] e^{i<\omega_{(p)} \mid \omega_{(p)}>}=1 .
$$

Note that both the inner product (2.3) and hence the functional measure in (2.4) depend explicitly on the metric on $M$. Even for compact closed $M$, the functional measure (2.4) is formally infinite and requires regularization.

One can use the invariance of (2.4) to determine how the functional measure transforms under the reparametrization (2.1). Using (2.1) and the orthogonality of the Hodge decomposition with respect to the inner product (2.3) one can show that:

$$
\begin{aligned}
<\omega_{(p)} \mid \omega_{(p)}> & =<h_{(p)}\left|h_{(p)}>+<d \alpha_{(p-1)}\right| d \alpha_{(p-1)}>+<\delta \beta_{(p+1)} \mid \delta \beta_{(p+1)}> \\
& =<h_{(p)}\left|h_{(p)}>+<\alpha_{(p-1)}\right|(\delta d) \alpha_{(p-1)}>+<\beta_{(p+1)} \mid(d \delta) \beta_{(p+1)}>,(2.5
\end{aligned}
$$

where we have used the identities [17]:

$$
<d \alpha|d \alpha>=<\alpha|(\delta d) \alpha>
$$

and

$$
<\delta \beta|\delta \beta>=<\beta|(d \delta) \beta>.
$$

Since, for any linear operator $O$, one has:

$$
\int \mathcal{D} \mu\left[\omega_{(p)}\right] \exp i<\omega_{(p)} \mid O \omega_{(p)}>=\operatorname{det}^{-\frac{1}{2}}(O),
$$

(2.4) and (2.5) imply that

$$
\mathcal{D} \mu\left[\omega_{(p)}\right]=\mathcal{D} \mu\left[h_{(p)}\right] \mathcal{D} \mu\left[\alpha_{(p-1)}\right] \mathcal{D} \mu\left[\beta_{(p+1)}\right] \operatorname{det}^{\frac{1}{2}}(\delta d)_{(p-1)} \operatorname{det}^{\frac{1}{2}}(d \delta)_{(p+1)} .
$$


Note that uniqueness of the Hodge decomposition requires that the zero modes of the relevant operators are excluded from the parametrization so that the Jacobian of the transformation is non-singular (when regulated appropriately).

\subsection{Abelian Chern-Simons Theory}

The classical action for an abelian Chern-Simons theory,

$$
S=\int_{M} A \wedge d A,
$$

is invariant (up to a total divergence) under the gauge transformation:

$$
A \rightarrow A+d \Lambda
$$

We wish to compute the partition function for the theory:

$$
Z:=\int \frac{\mathcal{D} \mu[A]}{V_{G}} e^{i S[A]}
$$

In the above $V_{G}$ denotes the volume of the group of gauge transformations in (2.10), which must be factored out of the partition function in order to guarantee that the integration is performed only over physically distinct gauge fields. The normal method for handling this is to add a lagrange multiplier term enforcing a gauge fixing condition to the classical action, and then to use the Fadeev-Popov ansatz, or its generalizations to add compensating ghost terms to the functional integral in order to guarantee that only physical modes contribute[16]. Alternatively, as we shall now demonstrate for the simple case of abelian Chern-Simons theory, the same results can be obtained by using the Hodge decomposition to parametrize the potential $A$ in terms of its gauge invariant, and gauge dependent parts, so that the volume of the group of gauge transformations can be explicitly factored out, leaving a functional integral over gauge invariant modes only.

We now transform the integration variables:

$$
A \rightarrow \alpha, \beta, h
$$

where $\alpha, \beta, h$ parameterize respectively the exact, co-exact, and harmonic parts of the connection A. Taking into account the Jacobian (2.5) we get:

$$
Z=\int \frac{\mathcal{D} \mu[\alpha] \mathcal{D} \mu[\beta] \mathcal{D} \mu[h]}{V_{G}} \operatorname{det}^{\frac{1}{2}} \Delta_{(0)} \operatorname{det}^{\frac{1}{2}}(d \delta)_{(2)} e^{i S}
$$


where we have used the fact that $\Delta_{(0)}=(\delta d)_{(0)}$. In terms of this parametrization, it is clear that the volume of the gauge group is simply

$$
V_{G}=\int \mathcal{D} \mu[\alpha]
$$

while the classical action functional becomes, after integrating by parts, using the properties (2.2) of $h$ and the nilpotency of the exterior derivative operators, and dropping surface terms:

$$
S=-<\beta \mid(* \delta d \delta) \beta>
$$

Note that $S$ depends only the co-exact (transverse) part of $A$. Using (2.13) and integrating over $\beta$ yields:

$$
Z=\int \mathcal{D} \mu[h] \operatorname{det}^{-\frac{1}{2}}(* \delta d \delta)_{(2)} \operatorname{det}^{\frac{1}{2}} \Delta_{(0)} \operatorname{det}^{\frac{1}{2}}(d \delta)_{(2)} .
$$

In the Appendix we prove that

$$
\operatorname{det}(* \delta d \delta)_{(2)}=\operatorname{det}^{\frac{1}{2}}((d \delta d)(\delta d \delta))_{(2)}=\operatorname{det}^{\frac{3}{2}}(d \delta)_{(2)} .
$$

Using the identity

$$
\operatorname{det}(\delta d)_{(p)}=\operatorname{det}(d \delta)_{(n-p)},
$$

which is a consequence of Hodge duality, it follows that

$$
Z=\int \mathcal{D} \mu[h] \operatorname{det}^{-\frac{3}{4}} \Delta_{(1)}^{T} \operatorname{det}^{\frac{1}{2}} \Delta_{(0)} \operatorname{det}^{\frac{1}{2}} \Delta_{(1)}^{T} .
$$

The operator $\Delta_{(p)}^{T}$ is the transverse part of the laplacian acting on $p$-forms:

$$
\Delta_{(p)}^{T}:=(\delta d)_{(p)}
$$

Applying identity (A.3) proved in the Appendix, we have $\operatorname{det} \Delta_{(1)}^{T}=\operatorname{det} \Delta_{(1)} / \operatorname{det} \Delta_{(0)}$ and hence

$$
Z=\int \mathcal{D} \mu[h] \operatorname{det}^{-\frac{1}{4}} \Delta_{(1)} \operatorname{det}^{\frac{3}{4}} \Delta_{(0)}
$$

The space of harmonic forms (of any order) consists of a finite set. Hence, the integration over harmonic forms in the above is a simple sum. We recognize the integrand in the above as the inverse of the square root of the Ray-Singer torsion of the de Rham cohomology of $M[2,13]$. 


\subsection{Abelian BF Theories}

The action for abelian BF theories is[2]

$$
S=\int B_{(n-2)} \wedge d A_{(1)}=-<B_{(n-2)} \mid * d A_{(1)}>,
$$

which is invariant under the gauge transformations:

$$
\begin{aligned}
B_{(n-2)} & \rightarrow B_{(n-2)}+d a_{(n-3)}, \\
A_{(1)} & \rightarrow A_{(1)}+d \alpha_{(0)} .
\end{aligned}
$$

The partition function for the theory is therefore:

$$
Z=\int \frac{\mathcal{D} \mu\left[B_{(n-2)}\right] \mathcal{D} \mu\left[A_{(1)}\right]}{V_{G}} \exp i S\left[B_{(n-2)}, A_{(1)}\right],
$$

where the volume of the gauge group $V_{G}$ will be evaluated below.

We now do a Hodge decomposition on the fields:

$$
\begin{gathered}
B_{(n-2)}=h_{(n-2)}+d a_{(n-3)}^{T}+\delta b_{(n-1)}^{L}, \\
A_{(1)}=h_{(1)}+d \alpha_{(0)}+\delta \beta_{(2)}^{L} .
\end{gathered}
$$

Note that we have added the superscripts $T, L$ to the Hodge parameters to remind ourselves that they are necessarily transverse or longitudinal, respectively.

In terms of the new parameters, the action is, up to a sign:

$$
\begin{aligned}
S & =-<B_{(n-2)} \mid * d A_{(1)}> \\
& =-<\delta b_{(n-1)}^{L} \mid * d \delta \beta_{(2)}^{L}> \\
& =-<b_{(n-1)}^{L} \mid(* \delta d \delta) \beta_{(2)}^{L}>.
\end{aligned}
$$

Taking the Jacobian into account, the partition function is

$$
\begin{aligned}
Z= & \int \mathcal{D} \mu\left[h_{(n-2)}\right] \mathcal{D} \mu\left[h_{(1)}\right] \frac{\mathcal{D} \mu\left[a_{(n-3)}^{T}\right] \mathcal{D} \mu\left[\alpha_{(0)}\right]}{V_{G}} \mathcal{D} \mu\left[b_{(n-1)}^{L}\right] \mathcal{D} \mu\left[\beta_{(2)}^{L}\right] \\
& \times \operatorname{det}^{\frac{1}{2}}(\delta d)_{(n-3)} \operatorname{det}^{\frac{1}{2}}(d \delta)_{(n-1)} \operatorname{det}^{\frac{1}{2}} \Delta_{(0)} \operatorname{det}^{\frac{1}{2}}(d \delta)_{(2)} \quad e^{i S} .
\end{aligned}
$$

Integrating over $b_{(n-1))}^{L}$ yields the delta function: $\delta\left(* \delta d \delta \beta_{(2)}^{L}\right)$ so that, using the functional analogue of

$$
\int d x \delta(f(x))=\frac{1}{f^{\prime}(0)}
$$


the integral over $\beta_{(2)}^{L}$ yields:

$$
\begin{aligned}
Z= & \int \mathcal{D} \mu\left[h_{(n-2)}\right] \mathcal{D} \mu\left[h_{(1)}\right] \frac{\mathcal{D} \mu\left[a_{(n-3)}^{T}\right] \mathcal{D} \mu\left[\alpha_{(0)}\right]}{V_{G}} \operatorname{det}^{\frac{1}{2}}(\delta d)_{(n-3)} \operatorname{det}^{\frac{1}{2}}(d \delta)_{(n-1)} \\
& \times \operatorname{det}^{\frac{1}{2}} \Delta_{(0)} \operatorname{det}^{\frac{1}{2}}(d \delta)_{(2)} \operatorname{det}^{-1}(* \delta d \delta)_{(2)} .
\end{aligned}
$$

By using the identities (A.1) and

$$
\operatorname{det}(d \delta)_{(n-1)}=\operatorname{det}(\delta d)_{(n-2)}=\operatorname{det}(d \delta)_{(2)},
$$

which follow from (A.2), we get

$$
\begin{aligned}
Z= & \int \mathcal{D} \mu\left[h_{(n-2)}\right] \mathcal{D} \mu\left[h_{(1)}\right] \frac{\mathcal{D} \mu\left[a_{(n-3)}^{T}\right] \mathcal{D} \mu\left[\alpha_{(0)}\right]}{V_{G}} \\
& \times \operatorname{det}^{\frac{1}{2}}(\delta d)_{(n-3)} \operatorname{det}^{-\frac{1}{2}}(\delta d)_{(n-2)} \operatorname{det}^{\frac{1}{2}}(\delta d)_{(0)}
\end{aligned}
$$

The final step in the calculation is to evaluate $V_{G}$. From Equations (2.21) it is clear that:

$$
V_{G}=V_{B_{(n-2)}} \times V_{A_{(1)}},
$$

where, as in the case of Chern-Simons theory:

$$
V_{A_{(1)}}=\int \mathcal{D} \mu\left[\alpha_{(0)}\right]
$$

By analogy, one would be tempted to assume that:

$$
V_{B_{(n-2)}}=\int \mathcal{D} \mu\left[a_{(n-3)}^{T}\right]
$$

However, this is not consistent with the Faddeev-Popov ansatz for gauge fixing, which in turn is justified by a Hamiltonian analysis[16]. In particular, (2.31) would result in the wrong mode count for $\mathrm{BF}$ theories and also for dynamical theories with reducible symmetries[19].

The Faddeev-Popov ansatz can nonetheless be given a very simple geometrical interpretation in terms of the considerations above, and the calculation of $V_{B_{(n-2)}}$ turns out to be remarkably simple. Gauge fixing via Faddeev-Popov is equivalent to considering $V_{B_{(n-2)}}$ to be the volume of all possible (non-harmonic) $(n-3)$ forms, $a_{(n-3)}$, modulo the group of transformations on $a_{(n-3)}$ that leave $d a_{(n-3)}$ invariant[16]. Since the ghost-for-ghost prescription[23] treats these residual transformations in essentially the same way as the 
original transformations (i.e. it adds gauge fixing terms, compensating ghosts, etc.) it becomes apparent that

$$
V_{B_{(n-2)}}=\frac{\int \mathcal{D} \mu\left[a_{(n-3)}\right]}{V_{B_{(n-3)}}} .
$$

This equation can be easily iterated to yield ${ }^{1}$ :

$$
V_{B_{(n-2)}}=\prod_{i=3}^{n}\left[\int \mathcal{D} \mu\left[a_{(n-i)}\right]\right]^{(-1)^{(i+1)}} .
$$

We now express

$$
a_{(n-i)}=a_{(n-i)}^{T}+d a_{(n-i-1)}^{T}
$$

where we have ignored harmonics. This is because they do not contribute directly to the gauge group and can be factored out of the integral over $a_{(n-3)}$ without introducing any Jacobians. The decomposition (2.34) yields the following functional measure:

$$
\mathcal{D} \mu\left[a_{(n-i)}\right]=\mathcal{D} \mu\left[a_{(n-i)}^{T}\right] \mathcal{D} \mu\left[a_{(n-i-1)}^{T}\right] \operatorname{det}^{\frac{1}{2}}(\delta d)_{(n-i-1)}
$$

Inserting (2.35) into (2.33) yields the following simple expression:

$$
V_{B_{(n-2)}}=\int \mathcal{D} \mu\left[a_{(n-3)}^{T}\right] \prod_{i=3}^{(n-1)} \operatorname{det}^{\frac{1}{2}(-1)^{i+1}}(\delta d)_{(n-i-1)},
$$

so that the partition function becomes:

$$
Z=\int \mathcal{D} \mu\left[h_{(n-2)}\right] \mathcal{D} \mu\left[h_{(1)}\right] \operatorname{det}^{\frac{1}{2}} \Delta_{(0)} \prod_{i=2}^{n} \operatorname{det}^{\frac{1}{2}(-1)^{i+1}}(\delta d)_{(n-i)}
$$

It is straightforward to relate (2.37) to the expressions obtained previously by Schwarz[13] and by Blau and Thompson[2]. It is useful to write out the product in (2.37) explicitly:

$$
\begin{gathered}
Z=\int \mathcal{D} \mu\left[h_{(n-2)}\right] \mathcal{D} \mu\left[h_{(1)}\right] \operatorname{det}^{\frac{1}{2}} \Delta_{(0)} \operatorname{det}^{-\frac{1}{2}}(\delta d)_{(n-2)} \operatorname{det}^{\frac{1}{2}}(\delta d)_{(n-3)} \ldots \\
\ldots \operatorname{det}^{\frac{1}{2}(-1)^{n-1}}(\delta d)_{(2)} \operatorname{det}^{\frac{1}{2}(-1)^{n}}(\delta d)_{(1)} \operatorname{det}^{\frac{1}{2}(-1)^{n+1}} \Delta_{(0)} .
\end{gathered}
$$

In even dimensions, $n=2 m$, the identity (A.12) clearly guarantees the pairwise cancellation of all the determinants in the above expression, so that the partition function is unity.

\footnotetext{
1 A similar result was obtained by Obukhov[20] for antisymmetric tensor field theory.
} 
In odd dimensions, $n=2 m+1$, the corresponding factors have exponents with the same sign, and do not cancel. Instead, one obtains:

$$
Z=\int \mathcal{D} \mu\left[h_{(n-2)}\right] \mathcal{D} \mu\left[h_{(1)}\right] \operatorname{det}^{\frac{1}{2}(-1)^{m}}(\delta d)_{m} \prod_{i=0}^{m-1} \operatorname{det}^{(-1)^{i}}(\delta d)_{i}
$$

One can now use (A.3) to write this in terms of Laplacians:

$$
Z=\int \mathcal{D} \mu\left[h_{(n-2)}\right] \mathcal{D} \mu\left[h_{(1)}\right] \prod_{j=0}^{m} \operatorname{det}^{\nu_{j}} \Delta_{(j)},
$$

where $\nu_{j}:=(-1)^{j}\left(m-j+\frac{1}{2}\right)$. The expression for the partition function given by Blau and Thompson[2], for example, is considerably more complicated:

$$
Z=\int \mathcal{D} \mu\left[h_{(n-2)}\right] \mathcal{D} \mu\left[h_{(1)}\right] \operatorname{det}^{\tilde{\nu}_{0}} \Delta_{(1)} \operatorname{det}^{\tilde{\nu}_{1}} \Delta_{(0)} \prod_{j=0}^{2 m-1} \operatorname{det}^{\tilde{\nu}_{j}} \Delta_{(2 m-1-j)}
$$

where $\tilde{\nu}_{j}:=(-1)^{j+1}(2 j+1) / 4$. However, by using Hodge duality (A.11), it is easy to show that (2.41) reduces to (2.40) given above. 


\section{Non-Abelian Theories}

In this section, we will calculate the partition function for non-Abelian BF theories in n-dimensions, using techniques analoguous to those in the previous section. The action for these theories takes the form[2]:

$$
S[A, B]=\operatorname{Tr} \int_{M} B \wedge F(A)
$$

where $B$ is an $(n-2)$-form on $M$, taking its value in the Lie algebra $\mathcal{G}$ of a semisimple Lie group $G$ and $A$ is a $G$ - connection 1-form field on $M$. The curvature of $A, F(A)$ is defined by

$$
F(A):=d A+A \wedge A=d A+\frac{1}{2}[A, A] .
$$

The group $G$ generates the usual gauge transformations, under which the connection 1-form field $A$ transforms as

$$
\delta A=d_{A} \omega
$$

while the $\mathcal{G}$-valued $(n-2)$-form field $B$ transforms "vectorially"

$$
\delta B=[B, \omega]
$$

where $\omega$ is the gauge parameter, and hence is a $\mathcal{G}$-valued 0 -form field. The differential operator $d_{A}$ is the exterior covariant derivative operator with respect to the connection $A$ :

$$
d_{A} \omega:=d \omega+[A, \omega]
$$

and $[$,$] is the commutator of the \mathcal{G}$-valued forms.

Besides the " $\omega$-transformation", BF type topological field theories admit as a symmetry another transformation, namely

$$
\begin{gathered}
\delta A=0, \\
\delta B=d_{A} \tau,
\end{gathered}
$$

where $\tau$ is a $\mathcal{G}$-valued $(n-3)$-form field. 


\subsection{The Parametrization}

Consider any fixed connection $\tilde{A}$ with values in a lie algebra $\mathcal{G}$, such that $F[\tilde{A}]=0$; i.e. $\tilde{A}$ is flat. It is possible to do a generalized Hodge decomposition[18] for any $p$ - form $B_{(p)}$ that takes its values in the fibres of a flat vector bundle with connection $\tilde{A}$. To accomplish this, first define $d_{\tilde{A}}$, the covariant exterior derivative with respect to the flat connection $\tilde{A}$, by:

$$
d_{\tilde{A}} B_{(p)}:=d B_{(p)}+\left[\tilde{A}, B_{(p)}\right],
$$

acting on a $p$-forms $B_{(p)}$. The corresponding covariant co-exact derivative is:

$$
\delta_{\tilde{A}}:=(-1)^{n p+n+1} * d_{\tilde{A}} * .
$$

Since $\tilde{A}$ is flat, both $d_{\tilde{A}}$ and $\delta_{\tilde{A}}$ are nilpotent. The generalized Hodge decomposition of $B_{(p)}$ is:

$$
B=B^{H}+d_{\tilde{A}} \beta_{(n-3)}^{T}+\delta_{\tilde{A}} \gamma^{L},
$$

where the harmonic form $B^{H}$ satisfies

$$
\delta_{\tilde{A}} B^{H}=0=d_{\tilde{A}} B^{H},
$$

the transverse form field $\beta_{(n-3)}^{T}$ satisfies:

$$
\delta_{\tilde{A}} \beta_{(n-3)}^{T}=0 .
$$

Finally, the longitudinal field $\gamma^{L}$ satisfies

$$
d_{\tilde{A}} \gamma^{L}=0 .
$$

Given an arbitrary connection 1-form field $A$ (which in general is not flat), parametrize the connection 1-form field $A$ as follows:*:

$$
A=\tilde{A}+\delta_{\tilde{A}} \alpha^{L},
$$

where $\alpha^{L}$ is a longitudinal 2-form, and

$$
\tilde{A}:=U^{-1} \theta U+U^{-1} d U,
$$

\footnotetext{
* Elitzur et. al.[21] arrive at a similar expression for flat connections on two dimensional manifolds.
} 
now refers to the flat part of the connection $A$, so that

$$
F(\tilde{A}):=d \tilde{A}+\frac{1}{2}[\tilde{A}, \tilde{A}]=0 .
$$

Further, $\theta$ is a gauge invariant 1-form field such that

$$
d \theta+[\theta, \theta]=0
$$

and $U$ is a $G$-valued function over $M$. In terms of this decomposition, the curvature of $A$ is:

$$
F(A)=d_{\tilde{A}} \delta_{\tilde{A}} \alpha+\frac{1}{2}\left[\delta_{\tilde{A}} \alpha, \delta_{\tilde{A}} \alpha\right] .
$$

It is important to note the following:

i. The decomposition (3.13) is unique if and only if $F(A)=0$ implies that $\alpha^{L}=0$. We will henceforth assume this to be the case, and discuss the implications of non-trivial solutions in the concluding section;

ii. The condition (3.15) follows from the flatness of $\tilde{A}$.

In the next section, we will use this parameterization for the $A$ and $B$ fields in order to compute the partition function for non-abelian BF theories in $n$ dimensions.

\subsection{The Partition Function for Non-Abelian BF Theories}

The partition function for the quantum field theory is defined by:

$$
Z=\int \frac{\mathcal{D} \mu[A] \mathcal{D} \mu[B]}{V_{G}} \exp i S[A, B],
$$

where in general $\mathcal{D} \mu\left[\omega_{(p)}\right]$ denotes the functional measure on the space of Lie-algebra valued p-forms that normalizes the Gaussian functional integral:

$$
\int \mathcal{D} \mu\left[\omega_{(p)}\right] \exp i<\omega_{(p)} \mid \omega_{(p)}>=1
$$

with inner product now defined by

$$
<\omega_{(p)} \mid \omega_{(p)}>:=\operatorname{Tr} \int_{M} \omega_{(p)} \wedge * \omega_{(p)} .
$$

The trace above is with respect to the adjoint reppresentation of $G$.

We begin with the decomposition introduced in the first section. We first change variables from $(A, B) \rightarrow\left(\tilde{A}, \alpha^{L}, B^{H}, \gamma_{(n-1)}^{L}, \beta_{(n-3)}^{T}\right)$ The resulting form of the partition function is:

$$
Z=\int \mathcal{D} \mu[\tilde{A}] \mathcal{D} \mu\left[B^{h}\right] \mathcal{D} \mu\left[\beta^{T}\right] \frac{1}{V_{G}} J[\tilde{A}] Z^{\prime}[\tilde{A}]
$$


where $J[\tilde{A}]$ is the Jacobian of the transformation to the generalized Hodge components:

$$
\begin{aligned}
J[\tilde{A}] & :=\operatorname{det}^{\frac{1}{2}}\left(d_{\tilde{A}} \delta_{\tilde{A}}\right)_{(2)} \operatorname{det}^{\frac{1}{2}}\left(\delta_{\tilde{A}} d_{\tilde{A}}\right)_{(n-3)} \operatorname{det}^{\frac{1}{2}}\left(d_{\tilde{A}} \delta_{\tilde{A}}\right)_{(n-1)} \\
& =\operatorname{det}\left(d_{\tilde{A}} \delta_{\tilde{A}}\right)_{(2)} \operatorname{det}^{\frac{1}{2}}\left(\delta_{\tilde{A}} d_{\tilde{A}}\right)_{(n-3)} \cdot
\end{aligned}
$$

The last line in the above is obtained by using the identity (A.12). In Eq.(3.19) we have defined the functional integral:

$$
Z^{\prime}[\tilde{A}]:=\int \mathcal{D} \mu\left[\alpha^{L}\right] \mathcal{D} \mu\left[\gamma^{L}\right] \exp i<B^{H}+d_{\tilde{A}} \beta^{T}+\delta_{\tilde{A}} \gamma^{L} \mid d_{\tilde{A}} \delta_{\tilde{A}} \alpha^{L}+\left[\delta_{\tilde{A}} \alpha^{L}, \delta_{\tilde{A}} \alpha^{L}\right]>
$$

Although $Z^{\prime}$ appears to depend on $B_{H}$ and $\beta^{T}$ as well as $\tilde{A}$, we will now show that it depends only on $\tilde{A}$, hence justifying the notation.

The integration over $\alpha^{L}$ and $\gamma^{L}$ in (3.21) can be done as follows. First expand $\alpha^{L}$ in a complete, orthonormal basis of Lie-algebra-valued 2-form eigenmodes of the operator $\left(d_{\tilde{A}} \delta_{\tilde{A}}\right)_{(2)}$ :

$$
\alpha^{L}=\sum_{i} C_{i} \psi_{(2)}^{i}
$$

where

$$
\left(d_{\tilde{A}} \delta_{\tilde{A}}\right)_{(2)} \psi_{(2)}^{i}=\Lambda^{i} \psi_{(2)}^{i},
$$

and

$$
<\psi_{(2)}^{i} \mid \psi_{(2)}^{j}>=\delta^{i j}
$$

Similarly, expand:

$$
\gamma_{(n-1)}^{L}=\sum_{j} f_{i} \psi_{(n-1)}^{i}
$$

where the $\psi_{(n-1)}^{i}$ are normalized eigenmodes of $\left(d_{\tilde{A}} \delta_{\tilde{A}}\right)_{(n-1)}$, corresponding to eigenvalues $\lambda_{i}$.

We now prove that $\psi_{(n-1)}^{i}$ can be chosen so that

$$
* \delta_{\tilde{A}} \psi_{(n-1)}^{i}=\sqrt{\Lambda^{i}} \psi_{(2)}^{i} .
$$

First define $\tilde{\psi}_{(2)}^{i}:=-* \delta_{\tilde{A}} \psi_{(n-1)}^{i}$, and then evaluate

$$
\begin{aligned}
\left(d_{\tilde{A}} \delta_{\tilde{A}}\right)_{(2)} \tilde{\psi}_{(2)}^{i} & =d_{\tilde{A}} \delta_{\tilde{A}} * \delta_{\tilde{A}} \psi_{(n-1)}^{i} \\
& =(-1)^{n-1} * * d_{\tilde{A}} * * \delta_{\tilde{A}} * \delta_{\tilde{A}} \psi_{(n-1)}^{i}=-* \delta_{\tilde{A}} d_{\tilde{A}} \delta_{\tilde{A}} \psi_{(n-1)}^{i} \\
& =-\lambda^{i} * \delta_{\tilde{A}} \psi_{(n-1)}^{i}=\lambda^{i} \tilde{\psi}_{(2)}^{i} .
\end{aligned}
$$


This implies that $\tilde{\psi}_{(2)}^{i}$ are eigenmodes of $\left(d_{\tilde{A}} \delta_{\tilde{A}}\right)_{(2)}$, with eigenvalue $\lambda^{i}$. Similarly, one can show that $* \delta_{\tilde{A}} \psi_{(2)}^{i}$ are eigenmodes of $\left(d_{\tilde{A}} \delta_{\tilde{A}}\right)_{(n-1)}$ with eigenvalues $\Lambda^{i}$. This implies that one can always choose a basis in which $\psi_{(2)}^{i} \propto * \delta_{\tilde{A}} \psi_{(n-1)}$, with the constant of proportionality in (3.26) determined by the normalization conditions.

In terms of the above decompositions, $Z^{\prime}$ can be written:

$$
Z^{\prime}=\int \prod_{i} d C_{i} \exp i<B^{H}+d_{\tilde{A}} \beta^{T} \mid * F[A]>\int \prod_{j} d f_{j} \exp i f_{j} X^{j}(C),
$$

where we have defined

$$
X^{j}(C):=\sqrt{\Lambda_{j}}\left(C_{j} \Lambda_{j}+<\psi_{(2)}^{j} \mid \sum_{k, l}\left[C_{k} \psi_{(2)}^{k}, C_{l} \psi_{(2)}^{l}\right]>\right) .
$$

The integration over the coefficients $f_{i}$ can now be trivially performed to give a delta function: $\delta\left(X^{j}(C)\right)$. We now need to assume that $X^{j}(C)$ does not vanish for any values of $C_{i}$ other than $C_{i}=0$. This is the same as requiring that the operator $d_{A} \delta_{\tilde{A}}$ not have any zero eigenmodes, which was necessary in the previous section to ensure the uniqueness of the decomposition. Although this may not be true globally, we can restrict the region of integration to the neighbourhood of $C_{j}=0$, in which case it is plausible that the term quadratic in the $C$ 's can never cancel the term linear in $C$ 's. In this case, the functional integration over $\alpha^{L}$ has support only at $\alpha^{L}=0$, and, using (3.23), one obtains:

$$
Z^{\prime}[\tilde{A}]=\operatorname{det}^{-\frac{3}{2}}\left(d_{\tilde{A}} \delta_{\tilde{A}}\right)_{(2)},
$$

which depends only on $\tilde{A}$ as claimed. This is the crucial result of this paper, since it allows the rest of the partition function to be integrated in a straightforward fashion.

From (3.30), the partition function becomes

$$
Z=\int \mathcal{D} \mu[\tilde{A}] \mathcal{D} \mu\left[B^{H}\right] \mathcal{D} \mu\left[\beta^{T}\right] \frac{1}{V_{G}} \operatorname{det}^{-\frac{1}{2}}\left(d_{\tilde{A}} \delta_{\tilde{A}}\right)_{(2)} \operatorname{det}^{\frac{1}{2}}\left(\delta_{\tilde{A}} d_{\tilde{A}}\right)_{(n-3)} .
$$

We now parameterize $\tilde{A}$ according to (3.14). The Jacobian for this change of variable can be computed from the inner product $<\delta \tilde{A} \mid \delta \tilde{A}>$, where the variation $\delta \tilde{A}$ is

$$
\delta \tilde{A}=d_{\tilde{A}} \delta \lambda+U^{-1} \delta \theta U,
$$

with the variation $\delta \lambda:=U^{-1} \delta U$. The flatness of $\tilde{A}$ implies that (3.32) in fact coincides with the Hodge decomposition of its variation (which is after all a cotangent vector). Hence $U^{-1} \delta \theta U$ must be the harmonic part of $\delta \tilde{A}$, with the consequence that

$$
d_{\tilde{A}} \delta\left(U^{-1} \theta U\right)=0=\delta_{\tilde{A}} \delta\left(U^{-1} \theta U\right) .
$$


The result is that $<\delta \tilde{A} \mid \delta \tilde{A}>$ has no cross-terms in $\delta \lambda$ and $\delta \theta$ and hence the Jacobian for $\tilde{A} \rightarrow(U, \theta)$ is $\operatorname{det}^{\frac{1}{2}}\left(\delta_{\tilde{A}} d_{\tilde{A}}\right)_{(0)}$. The result for the partition function is

$$
Z=\int \mathcal{D} \mu[\theta] \mathcal{D} \mu\left[B^{H}\right] \mathcal{D} \mu[U] \mathcal{D} \mu\left[\beta^{T}\right] \frac{1}{V_{G}} \operatorname{det}^{\frac{1}{2}}\left(\delta_{\theta} d_{\theta}\right)_{(0)} \operatorname{det}^{-\frac{1}{2}}\left(d_{\theta} \delta_{\theta}\right)_{(2)} \operatorname{det}^{\frac{1}{2}}\left(\delta_{\theta} d_{\theta}\right)_{(n-3)} .
$$

where in the above $\mathcal{D} \mu[U]$ denotes the Haar measure on the gauge group, and we have used the gauge invariance of the functional determinants to replace the various covariant derivative operators with respect to $\tilde{A}$ by covariant derivative operators with respect to $\theta$.

It is convenient at this stage to define precisely what is meant by $V_{G}$, the "volume of the group of gauge transformations." This must be defined so as to eliminate the unphysical gauge modes in the functional integral. By performing the above reparametrizations we have effectively isolated these modes: they are contained in the group variables $U$ and the transverse $(n-3)$-forms $\beta^{T}$. As in the the abelian case, it would not be consistent with perturbation theory to simply eliminate the integrals over these variables. In complete analogy with the discussion in Section 2, the correct choice for $V_{G}$ is therefore:

$$
V_{G}=V_{A}^{(n-3)} V_{U}
$$

where

$$
V_{U}:=\int \mathcal{D} \mu[U]
$$

and

$$
V_{A}^{(n-3)}:=\int \frac{\mathcal{D} \mu\left[\beta^{(n-3)}\right]}{V_{A}^{(n-4)}} .
$$

In the above, it is important to notice that we functionally integrate over all the $(n-3)$ forms, and not just the transverse modes. We then divide by the volume of the orbits of gauge transformations acting on $(n-3)$-forms. The rest of the calculation, namely the integration over $U$ and $\beta^{T}$, proceeds as in section 2.3 after equation (2.31). The reason for this is that a small neighbourhood in the hypersurface (in configuration space) of flat connections has the same structure as the hypersurface of closed forms in the configuration space of abelian BF theory. The result is therefore identical in form to (2.37), namely:

$$
Z=\int \mathcal{D} \mu[\theta] \mathcal{D} \mu\left[B^{H}\right] \prod_{i=2}^{n} \operatorname{det}^{\frac{1}{2}(-1)^{i+1}}\left(\delta_{\theta} d_{\theta}\right)_{(n-i)} \quad \operatorname{det}^{\frac{1}{2}}\left(\delta_{\theta} d_{\theta}\right)_{(0)}
$$

The last factor in the above is square root of the functional determinant of the laplacian acting on 0 -forms with respect to the connection $\theta$, and it occurs in $Z$ only for odd-dimensional 
spacetimes. We can again use identities (A.3) and (A.12) to simplify (3.38) for odd $n$ to obtain:

$$
Z=\int \mathcal{D} \mu[\theta] \mathcal{D} \mu\left[B^{H}\right] \prod_{j=0}^{m} \operatorname{det}^{(-1)^{j}\left(m-j+\frac{1}{2}\right)} \Delta_{j}(\theta),
$$

where $\Delta_{j}(\theta)$ is the Laplacian $\left(\delta_{\theta} d_{\theta}+d_{\theta} \delta_{\theta}\right)_{j}$. In the case of even $n$, the same identities and (A.2) yield the result $Z=1$, i.e., the triviality of the Ray-Singer torsion in even dimensional manifolds.

Note that the integrand in (3.39) is in general a function of the $\theta$, the coordinates in the space of equivalence classes (under gauge transformations) of flat connections on $M$. Hence, the integration is over the bundle $\mathcal{N}$ to the moduli space $\mathcal{M}$ over $M$, with fibers isomorphic to the cohomology space $H^{(n-2)}(M)$. In particular, consider the case $n=3$, with the gauge group $G$ the Lorentz group $\mathrm{SO}(2,1)$, so that the BF theory is general relativity in $2+1$ dimensions. In this case the space $\mathcal{N}$ is just the cotangent bundle to $\mathcal{M}[15]$.

For $n=3$, our result corresponds to the expression obtained by Witten in Reference 15: One simply uses the result (A.11) to obtain

$$
Z=\int \mathcal{D} \mu[\theta] \mathcal{D} \mu\left[B^{H}\right] \operatorname{det}^{-\frac{1}{2}} \Delta_{(1)}(\theta) \operatorname{det}^{\frac{3}{2}} \Delta_{(0)}(\theta),
$$

and then notes that the functional determinant of Witten's operator $L_{-}$can be written as

$$
\operatorname{det}\left(L_{-}\right)=\operatorname{det}^{\frac{1}{2}} \Delta_{(1)}(\theta) \operatorname{det}^{\frac{1}{2}} \Delta_{(0)}(\theta)
$$




\section{Conclusions}

We have expressed the partition function for non-abelian and abelian BF type topological field theories (and for abelian Chern-Simons theory) in terms of functional determinants of the appropriate differential operators. This was achieved by introducing appropriate parameterizations of the fields, suggested by the geometry of the configuration spaces in question. Our procedure avoided many of the complications (in particular the introduction of non-physical ghost fields and ghosts for ghosts) associated with the technology of gauge-fixing and provided a simple expression for the volume of the gauge orbits for both abelian and non-Abelian B-F theories (cf. Eq.(2.36)).

In the Abelian case, our parameterizations consist of precisely the Hodge decompositions for the fields, as suggested by Horowitz and Srednicki[12] and by Blau and Thompson[2], and our results agree with those obtained in the latter paper and by Schwarz in [13], though we presented them in a somewhat simpler form. In addition, we were able to derive the identities necessary to prove the triviality of the partition function directly from the properties of the Hodge decomposition, without using functional integrals, as was done in Ref.2.

For non-abelian theories, the (Lie algebra-valued) vector field $B$ was parameterized by its Hodge decomposition with respect to the flat connection $\tilde{A}$. Our parameterization of the connection 1-form field $A$ is rather more speculative, in the sense that we must remain in a neighbourhood of the flat connection $\tilde{A}$ sufficiently small so that the solutions of $F(A)=0$ are all at most gauge transformations of $\tilde{A}$. In this case, we were able to explicitly compute the partition function for any dimension of the spacetime manifold in terms of functional determinants of twisted Laplacians with respect to the flat connections $\tilde{A}$. In other words, the partition function (3.39) is an integral over some bundle over the moduli space of flat connections of an integrand which is essentially the Ray-Singer torsion with respect to that flat connection. ${ }^{1}$ We showed that, as expected, the partition function is trivial in the case of even dimensional spacetimes. For three dimensional spacetime our partition function agrees with that obtained by Witten[15]. As far as we know ours is the first explicit result for dimensions greater than four.

We are currently working on the extension of our method to calculate partition functions for super BF theories and other topological field theories. Furthermore, we are attempting to explicate the structure of configuration space in more detail in order to improve the applicability of our parameterization.

1 It is perhaps worth noting that this result can be reformulated as a statement that the semi-classical approximation in such theories is exact. 


\section{ACKNOWLEDGEMENTS}

We wish to thank S. Carlip, B. Dolan, P. Kelly and D.J. Toms for useful suggestions and discussion. J.G. is grateful to the Winnipeg Institute of Theoretical Physics for its kind hospitality and support during the latter stages of this work. We also wish to acknowledge the partial support of the Natural Sciences and Engineering Research Council of Canada. 


\section{APPENDIX}

We now prove three important identities used in the text. In this section the differential operators $d$ and $\delta$ refer to the exterior covariant derivative with respect to any flat connection, $\tilde{A}$, and its co-derivative, respectively, acting on lie-algebra valued forms. The results are of course equally valid for the Abelian counterparts of these operators used in Section 2. For simplicity, however, we have suppressed the subscript $\tilde{A}$. We shall prove:

$$
\begin{gathered}
\operatorname{det}[* \delta d \delta]=\operatorname{det}^{\frac{1}{2}}[d \delta d * * \delta d \delta]=\operatorname{det}^{\frac{3}{2}}[d \delta] ; \\
\operatorname{det}(d \delta)_{(p)}=\operatorname{det}(\delta d)_{(p-1)} ; \\
\operatorname{det} \Delta_{(p)}=\operatorname{det}(\delta d)_{(p)} \operatorname{det}(\delta d)_{(p-1)} .
\end{gathered}
$$

Note that on the right hand side of (A.1), we have dropped a field independent phase.

First consider an invertible operator $O$ that maps the space of $p$-forms onto the space of $p^{\prime}$-forms. Given an inner product on both the space of $p$ and $p^{\prime}$ forms, the adjoint $O^{\dagger}$ of $O$ is defined by:

$$
<O \omega_{(p)}\left|\omega_{(p)}^{\prime}>=<\omega_{(p)}\right| O^{\dagger} \omega_{(p)}^{\prime}>
$$

In general the determinant of such an operator is ambiguous, even if it is invertible, because one needs to define separate bases for the space of $p$-forms and the space of $p^{\prime}$-forms, so that invariance is $\operatorname{lost}^{1}$. In the present case, however, the determinant in question is essentially defined by the path integral that we need to evaluate, namely (cf. Eq.(2.26)):

$$
\operatorname{det}^{-1}(O) \equiv \int \mathcal{D} \mu\left[\omega_{(p)}\right] \mathcal{D} \mu\left[\omega_{\left(p^{\prime}\right)}\right] \exp i<O \omega_{(p)} \mid \omega_{\left(p^{\prime}\right)}>
$$

Since $O$ is assumed invertible, we can parametrize the space of $p^{\prime}$-forms in terms of $p$-forms:

$$
\omega_{\left(p^{\prime}\right)}=O \omega_{(p)}^{\prime}
$$

The resulting Jacobian, $\mathrm{J}(\mathrm{O})$, can be evaluated by demanding that:

$$
\int \mathcal{D} \mu\left[\omega_{(p)}^{\prime}\right] \exp i<O \omega_{(p)}^{\prime}\left|O \omega_{(p)}^{\prime}>=\int \mathcal{D} \mu\left[\omega_{\left(p^{\prime}\right)}\right] J^{-1}(O)<\omega_{\left(p^{\prime}\right)}\right| \omega_{\left(p^{\prime}\right)}>
$$

After performing the two Gaussians in the above expression, this yields:

$$
J(O)=\operatorname{det}^{\frac{1}{2}}\left(O^{\dagger} O\right)
$$

1 We are grateful to Steve Carlip for bringing this fact to our attention 
Performing the change of variables, and inserting this Jacobian in the functional integral (A.5) gives:

$$
\begin{aligned}
\operatorname{det}^{-1}(O) & =\int \mathcal{D} \mu\left[\omega_{(p)}\right] \mathcal{D} \mu\left[\omega_{(p)}^{\prime}\right] \operatorname{det}^{\frac{1}{2}}\left(O^{\dagger} O\right) \exp i<O \omega_{(p)} \mid O \omega_{(p)}^{\prime}> \\
& =\int \mathcal{D} \mu\left[\omega_{(p)}\right] \mathcal{D} \mu\left[\omega_{(p)}^{\prime}\right] \operatorname{det}^{\frac{1}{2}}\left(O^{\dagger} O\right) \exp i<\omega_{(p)} \mid O^{\dagger} O \omega_{(p)}^{\prime}> \\
& =\operatorname{det}^{-\frac{1}{2}}\left(O^{\dagger} O\right) .
\end{aligned}
$$

(A.7) is of course consistent with the definition of $\operatorname{det}(O)$, used in previous work $[2,12,13]$.

If we now consider the operator $O=* \delta d \delta$, then it is easy to verify that the adjoint is $O^{\dagger}=d \delta d *$, and (A.1) follows.

To prove the identity (A.2), note that if $\omega_{(p)}$ is an eigenmode of $(d \delta)_{(p)}$ with eigenvalue $\lambda$, then $\delta \omega_{(p)}$, which is a $(p-1)$-form is an eigenmode of $(\delta d)_{(p-1)}$ with the same eigenvalue $\lambda$. But all the non- zero eigenmodes of $(\delta d)_{(p-1)}$ must be co-exact, and hence all of its eigenmodes must be of the form $\delta \omega_{(p)}$. Thus the operators in question in (A.2) have the same eigenmodes and eigenvalues, and the result follows. Finally, to prove (A.3), we use the orthogonality of the decomposition

$$
\Delta_{(p)}=(\delta d+d \delta)_{(p)},
$$

to prove

$$
\operatorname{det} \Delta_{(p)}=\operatorname{det}(\delta d)_{(p)} \quad \operatorname{det}(d \delta)_{(p)},
$$

and then use (A.2) to get (A.3).

We also note that (A.3) can be solved for $\operatorname{det}(\delta d)_{(p)}$ and iterated to yield

$$
\operatorname{det}(\delta d)_{(p)}=\prod_{i=0}^{p} \operatorname{det}^{(-1)^{i}} \Delta_{(p-i)} .
$$

Another useful identity, easily derived from the properties of Hodge duality is[12]:

$$
\operatorname{det} \Delta_{(p)}=\operatorname{det} \Delta_{(n-p)}
$$

Using this and (A.2) it is trivial to show that

$$
\operatorname{det}(d \delta)_{(p)}=\operatorname{det}(d \delta)_{(n-p+1)} .
$$




\section{REFERENCES}

1. E.Witten, Comm.Math.Phys. 117, 353 (1988).

2. M.Blau and G.Thompson, Ann. of Phys. 205,130 (1991).

3. A.Ashtekar, Lectures on Non-Perturbative Quantum Gravity, World Scientific, 1991.

4. E.Witten, Nuc. Phys. B 311, 46 (1988).

5. V. Moncrief, J. Math. Phys. 30,2907 (1989).

6. A. Hosoya and K. Nakao, Class. Quant. Grav. 7, 163 (1990).

7. S. Deser, R. Jackiw and G. t'Hooft, Ann. of Phys. 152, 220 (1984).

8. A. Ashtekar, Class. Quant. Grav. 6, L185 (1989).

9. J. Nelson and T. Regge, Nuc. Phys. B 328, (1989); S.P. Martin, Nuc. Phys. B 327, 178 (1990); S. Carlip, Phys. Rev. D 42, 2647 (1990).

10. S. Carlip, Class. Quant. Grav. 8, 5 (1990).

11. G.T. Horowitz, Comm. Math. Phys. 125, 417 (1989).

12. G.T. Horowitz and M. Srednicki, Comm. Math. Phys. 130, 83 (1990).

13. A.S. Schwarz, Lett. Math. Phys. 2, 247 (1978); Comm. Math. Phys. 67, 1 (1979).

14. E. Witten, Comm. Math. Phys. 121, 351 (1989).

15. E. Witten, Nuc. Phys. B 323, 113 (1989).

16. See for example L.D. Faddeev and A.A. Slavnov, Gauge Fields: Introduction to Quantum Theory, Benjamin-Cumming, 1980; or M. Daniel and C. M. Viallet, Rev. Mod. Phys. 52, 175 (1980).

17. Y. Choquet-Bruhat, C. DeWitt-Morette and M. Dillard- Bleick, Analysis, Manifolds and Physics, Revised Edition, North-Holland (1982).

18. T. Eguchi, P.B. Gilkey and A.J. Hanson, Phys. Reports 66, 213 (1980).

19. P.K. Townsend, Phys. Lett. B 88, 97 (1979); W. Siegel, Phys. Lett. B 93, 170 (1980).

20 Yu. N. Obukhov, Phys. Letts. 109B, 195 (1982).

21. S. Elitzur et. al., Nuc. Phys. B 326, 108 (1989). 